\title{
EL PROFESORADO Y SUS DISCURSOS EN RELACIÓN AL GÉNERO EN UNA ESCUELA PÚBLICA
}

\author{
Grawen OJEDA URRA ${ }^{1}$
}

\begin{abstract}
Resumen
El artículo se centra en el discurso como generador de formas de pensamiento de género, en una escuela pública de Chile desde la práctica docente y qué formas utilizan éstos, para reforzar posturas hegemónicas. El estudio aporta indicadores claves, reflejados en la desigualdad de género que invisibilizan lo femenino. Sin embargo, se reconocen algunas prácticas pedagógicas que privilegia la igualdad hacia los estudiantes.
\end{abstract}

Palabras clave: discurso de género, práctica docente, análisis del discurso, igualdad de género, hegemonía de lo masculino.

\section{Resumé}

L'article se concentre sur le discours comme un générateur de formes de genre de pensé, dans une école publique au Chili de l'enseignement pratique et ce qui fait qu'ils utilisent pour renforcer les positions hégémoniques. L'étude fournit des indicateurs clés, reflétés dans l'inégalité de genre féminin invisible. Toutefois, certaines pratiques pédagogiques qui favorise l'égalité de reconnaître les étudiants.

Mots-clés: discours sexe, pratique l'enseignement, analyse du discours, l'égalité des sexes, l'hégémonie masculine.

\section{Abstract}

The article focuses on speech, as a generator of forms of gender thinking in a public school in Chile from teaching practice and what forms they use to reinforce hegemonic

\footnotetext{
${ }^{1}$ Estudiante doctorado del consorcio: (Universidad Católica del Maule, Universidad Católica de la Santísima Concepción, Universidad Católica de Temuco y Universidad del Bío-Bío). grawenojeda@gmail.com
}

Fecha de recepción del artículo: Mayo 2016

Fecha de evaluación: Junio 2016 
positions. The study provides key indicators, reflected in gender inequality which invisible feminine. However, some pedagogical practices that favor equality to recognize students.

Keywords: gender discourse, teaching practice, discourse analysis, gender equality, male hegemony.

\section{Introducción}

Algunos de los significados que imperan en la cultura de género provocan situaciones sociales, personales y educativas no gratas. En el marco de la convivencia, éstas muchas veces desencadenan situaciones de bullying, la violencia de género, discriminación y exclusión entre otras, que dañan los derechos y la dignidad de quienes las padecen. En este contexto, los docentes por su parte, resultan ser un elemento central tanto por su impacto en el aprendizaje de los estudiantes como en la cultura escolar, a través de sus prácticas de saberes y discursos, los que han adquirido a través de sus experiencias de vida durante su trayectoria profesional. Desde esta perspectiva el quehacer pedagógico asume una doble intención, por un lado es portadora de diferencias entre género y por otra es creadora de dinámicas educativas superadoras de la desigualdad. Estas dimensiones inciden en los planteamientos pedagógicos y didácticos que realiza el profesorado (Sánchez, 2015). Sin embargo, se puede dar el caso que desarrolle la capacidad de generar y desarrollar estrategias y formas innovadoras de actuación, así como de intencionar discursos que fomenten un giro de prácticas hacia la igualdad.

La investigación sobre género y discurso, ha tenido un tratamiento divergente y plural en los últimos años. En la actualidad, esta temática resulta transversal a multitud de disciplinas y corrientes científicas que profundizan en esta temática reflejando las realidades que se suceden en diversos contextos. Su desarrollo está vinculado a una diversidad de procedimientos metodológicos que intentan dar respuesta a las interrogantes que surgen de las problemáticas detectadas por los investigadores (Cortés, 2007).

En su planteamiento entre discurso y educación social Foucault (1970), considera que existen grandes hendiduras. En educación, por más que sea, de derecho, es decir, instrumento gracias al cual todo individuo en una sociedad puede acceder, independiente del tipo de discurso que utilice, está condicionado a lo que permite y en lo que impide, en las líneas marcadas por las diferencias, las oposiciones y las luchas antagónicas como las diferencias de clase y de pensamiento social.

Con el propósito de responder a las problemáticas de género presentes en el discurso de los docentes del sistema educativo, el objetivo del presente estudio es analizar los discursos de los profesores y profesoras de una escuela pública, a través de los cuales se pretende saber la percepción respecto a la igualdad de género en función de sus prácticas pedagógicas. El estudio propone explorar los discursos de profesores y profesoras como reforzamiento de la diferencia de género en la escuela en el contexto del ejercicio docente. Se realiza desde un enfoque cualitativo y los datos son recolectados a través de la técnica de la entrevista semi - estructurada a 6 docentes y enmarcado en el diseño de estudio de casos.

Para acceder al discurso se utilizó el análisis de contenido, desde la cual emergen 2 categorías centrales de análisis: igualdad de género y hegemonía de lo masculino. A través de este planteamiento se espera aportar con información a la realidad educativa 
que se desprende del discurso sobre las prácticas pedagógicas de los docentes de una escuela pública en la región del Maule ubicada en la zona centro sur de Chile.

\section{Relación entre discurso y género}

West, Lazar \& Kramarae, (2000), sostienen que hace más de dos décadas el género y el discurso han sido campo de investigación, los que actualmente abordan distintas problemáticas que subyacen a estos dos grandes temas. El interés por investigar en este ámbito surge de la relación entre el género y el lenguaje, (Cameron, 1992). Jiménez (2007), considera que los analistas del discurso de género establecen el lenguaje como un ámbito de construcción del género. Asimismo, plantean que la construcción social del género, no es neutral, sino que está vinculada a las relaciones de poder institucionalizadas al interior de la sociedad. En esta línea, el discurso está siempre inmerso en un determinado contexto social. En este sentido Van Dijk (2005), establece que el discurso no puede ni debe estudiarse separadamente de sus variadas clases de contextos.

Respecto a la noción de discurso, la diversidad de antecedentes dificultan la formulación de una definición unívoca y uniforme del concepto, (Garay, Iñiguez \& Martínez, 2005). Los investigadores Gilbert \& Mulkay (1984), se refieren al discurso como a todas las formas de hablar y escribir, en tanto para Foucault (1970), el discurso son aquellas prácticas lingüísticas más amplias que se desarrollan históricamente. En este sentido, el presente artículo, optará por la caracterización que define al discurso como un conjunto de prácticas lingüísticas que mantienen y promueven las relaciones sociales. En tanto, el análisis del discurso consiste en estudiar cómo las prácticas discursivas que sostienen y promueven las relaciones sociales en determinados contextos internacionales (Iñíguez \& Antaki 1994).

Retomando la relación discurso y género, este enfoque se debe en gran parte a los aportes de Lakoff (1975), quien describió el lenguaje característico de las mujeres como diferente al de los hombres en relación al; léxico, la sintaxis y la pragmática. Según este autor, las mujeres usan mucho más mitigadores (por ejemplo, pienso y creo) y calificativos innecesarios (por ejemplo; muy hermoso, muy bellos, realmente feliz). Dentro de un estudio de género, este lenguaje hace ver el habla de las mujeres sin poder y con carencia de decisión; y por ello las descalifica de posiciones de poder y autoridad. (Lakoff, 1975).

\section{Discurso y género en el contexto educativo}

Los discursos del profesorado que caracterizan el quehacer docente permanecen impregnados de significados de género que impactan en la cultura escolar, convirtiéndose en la base y guía de la acción que se reproduce a diario. Esto se explica en la importancia del rol docente como elemento central en la escuela, lo que se manifiesta a través de su puesta en práctica de saberes y discursos que han adquirido en su propia trayectoria profesional y que parten de su experiencia de vida (West, Lazar \& Kramarae, 2000). Asimismo, puede desarrollar la capacidad profesional de generar estrategias y formas innovadoras de actuación, así como otros discursos que fomenten el cambio de este tipo de patrones culturales de género (Jiménez, 2007). Por lo tanto la práctica docente asume en este trabajo una doble vía, por una parte es portadora de la carga de género y por otra es creadora de dinámicas educativas que intentan superar la desigualdad. 
- Lo que los organismos internacionales dicen sobre el género en escuelas.

En el contexto social y en el marco de género, se puede sostener la permanente lucha por la igualdad, la cual puede ser más compleja que el género. Bolívar (2015), desde el paradigma de la igualdad, explica que todos los individuos deben optar y recibir lo mismo, sin distinción por ser diversos. Sin embargo, esta tarea se complejiza de tal forma, que es estudio constante de la disciplina de la Sociología la cual intenta dar respuesta a los fenómenos que relacionan e interactúan dentro de este concepto.

Organismos internacionales, como el Fondo de Población de las Naciones Unidas, insiste en la centralidad del género, en la defensa de los derechos humanos $\mathrm{y}$, el derecho a la igualdad, especialmente en educación, como un indicador fundamental para hablar; de desarrollo, ejercicio de derechos y efectividad de la igualdad (Vengas, 2010). La experiencia que enfrenta una niña y un niño en su establecimiento educacional, depende en gran medida de la cultura local, de las características de su familia, como también, de las estrategias que emplea el docente dentro de su práctica pedagógica.

- Estudios internacionales de género y discurso en las escuelas.

En un estudio realizado a profesoras que se desempeñan en la educación infantil, Da Silva \& Fanfa (2012), constatan el predominio de representaciones hegemónicas de feminidad y masculinidad, asociando lo femenino a lo delicado, mientras que lo masculino se enfoca a la virilidad y fortaleza.

En el contexto internacional España, por ejemplo, establece su Ley Orgánica 1/2004 del 28 de diciembre del año 2004, en su artículo 7 plantea que las Universidades deben incluir y fomentar en todos los ámbitos académicos la formación, docencia e investigación en igualdad de género y no discriminación. Por otra, los resultados de un estudio realizado entre los años 2012 - 2013 a 36 universidades españolas muestran una laguna formativa en educación para la igualdad de género (Bas, Pérez \& Vargas, 2014). Estos son claros ejemplos internacionales por desarrollar una preocupación e intentos fallecidos por resolver cuestiones de género al igual que en Chile.

Finco (2015), afirma que la escuela no es neutra, pero que, por el contrario, -participa levemente de la construcción de la identidad de género y lo realiza en forma desigual. Señala además que los profesionales de la educación se relacionan con infantes en guarderías y jardines infantiles y los temas se encuentran día a día en las prácticas pedagógicas. A pesar de esto, los temas de género todavía están lejos de las discusiones en los cursos de formación de profesoras y profesores. Vargas (2011), plantea que en países como Argentina, Chile, Estados Unidos, Uruguay, España y Costa Rica, se comparte un mismo sentido entre la educación y el género, donde la enseñanza es vista desde lo masculino, replicando modelos heredados de forma tradicional generacionalmente. En consecuencia, se puede sostener que la escuela es el espacio donde se articulan, fomentan y reproducen las desigualdades. Es probable que se deba a la persistencia de creencias estereotipadas y expectativas diferenciales de rol de género, aún presentes a nivel sociocultural y personal, por ejemplo, asociar a la mujer a la tarea de la maternidad y cuidado de los hijos. Sin embargo, por otra parte, existe el antecedente, que las niñas presentan predisposición a la construcción de una cultura de igualdad en la escuela (García, Rebolledo, García, Barragán \& Ruíz, 2010). Es decir, se revelan a quedar relegadas sólo a tareas de carácter doméstico.

En lo que respecta a la escuela secundaria desde donde se despliega la diversidad de identidades, siendo además, un lugar de encuentros y desencuentros, donde se combina la heterogeneidad, la diferenciación y la fragmentación induce a pensar, que el género 
interactúa en el sistema educativo. Esto obliga a plantearse cómo se construyen las relaciones sociales y se elaboran nuevos marcos interpretativos, que permitan explicar de qué forma opera la reproducción y transformación de estereotipos de género, en busca de igualdad de oportunidades educativas para los estudiantes (Parga, 2011).

Un aspecto investigado en relación al papel de los enseñantes y las actitudes que desarrollan hacia alumnos y alumnas, es la interacción en el aula entre maestras/os y alumna/os que refleja una aceptación inconsciente de los estereotipos sexistas (Subirats $\&$ Brullets, 1988). Esta investigación evidencia que las alumnas reciben menos tiempo de dedicación por parte del profesorado, además de un trato cualitativo distinto, en tanto se da mayor importancia a los planteamientos y tareas de los alumnos (Safilos \& Rosthschild, 1987).

Según Rodríguez (2011), las actitudes de los docentes forman parte de lo que se ha dado en denominar el currículum oculto, debido a que no son conscientes de las diferencias que establecen en la relación diaria con los niños y niñas de los establecimientos. Esto se grafica en el lamentable concepto que tienen los docentes sobre las alumnas, a pesar de conseguir como promedio un mayor éxito escolar, se decía que eran pasivas, dóciles, poco creativas y dependientes (Rodríguez, 2011). Todo ello se fundamenta en dos impresiones: la escasa participación de las niñas en los intercambios que se establecen en el aula y en el hecho de que busquen la protección y autoridad del docente para asegurar su espacio, donde las niñas manifiestan una conducta de ayuda a los otros y un mayor respeto a las normas. (CIDE, 1992).

Arnot, (2009), sostiene que existen creencias arraigadas en los docentes más tradicionales y conservadoras sobre las diferencias naturales entre alumnos y alumnas, justificadas por la tradición o por la experiencia personales del profesorado.

- La situación chilena.

En la realidad Chilena el tema de género, no fue una preocupación por parte de las autoridades educacionales imperantes entre los años 70 y 90 . Posterior a la década de los 80, se inicia la implementación de iniciativas específicamente en lo que respecta a la asistencia social, representada por la institucionalidad del Servicio Nacional de la Mujer. (Valdés, 2013). Sin embargo, como antecedente histórico la inquietud por incorporar a las mujeres en educación se basa en la presencia de la caridad, representado por los huérfanos y las órdenes religiosas, además de la necesidad de atender a las niñas que demandaban el proceso de enseñanza. (Eganaña, Núñez \& Salinas, 2003).

Con la llegada de la democracia en el año 1990, se asume un compromiso centrado en la superación de las desigualdades entre hombres y mujeres. Valdés (2013), plantea que ello se tradujo en la creación de una institucionalidad a cargo de esta tarea. Se realizaron modificaciones en la legislación, desarrollo de políticas y programas, a nivel sectorial y transversal, nacional, regional y local. Un ejemplo de ello es la creación del SERNAM (Servicio Nacional de la Mujer), a través del cual se han aplicado iniciativas sustentadas en la idea de transversalización del enfoque del género (Borries, 2012). No obstante, aún se mantiene en la sociedad chilena normas, prácticas y discursos institucionales que reproducen el lugar de subordinación de las mujeres y la vulneración de sus derechos. En el ámbito educativo, se ha avanzado en temas de acceso a la educación, donde ningún niño o niña debe quedar excluido de asistir a la escuela. Sin embargo, existe deserción escolar prematura, con patrones tradicionales de género: los hombres desertan por trabajo y las mujeres por funciones reproductivas y domésticas (Instituto Nacional de Derechos Humanos, 2012). 
En materia educativa el Ministerio de Educación realizó en el año 2013, Orientaciones para el Diseño e Implementación de un Programa en Sexualidad, Afectividad y Género y creó, además el Programa de Formación en Sexualidad, Afectividad y Género, con el fin de que los establecimientos educacionales recibieran las orientaciones y directrices para la implementación. Sin embargo, éstos dan escasos indicios de funcionamiento a inicios del año 2014.

- Estudios de género y discurso en escuelas en Chile.

$\mathrm{Al}$ comprender que las escuelas son un agente institucional, donde existe una serie de estructuras y prácticas que condicionan el proceso, es decir, que las escuelas producen y reproducen determinados regímenes, entre ellos, el género Madrid (2011), sostiene que se está frente a práctica permanente de desigualdad. Un régimen de género se refiere a que en las organizaciones existe un patrón de prácticas que define las conceptualizaciones de masculinidad y femineidad, jerarquizándolas en términos de prestigio y poder. Esto se expresa en la organización escolar, prácticas cotidianas, división de género en el trabajo, formas de ejercer autoridad y control (Madrid, 2011). Un estudio realizado el año 2011, -en 4 comunas del sur de Chile sostiene que la escuela chilena-, acentúa las diferencias entre niños y niñas de acuerdo a su clase social, mostrando que el proceso de discriminación por este factor en el sistema educativo se encuentra permeado y condicionado por la variable género, pudiendo constatar, por ejemplo, que las brechas entre hombres y mujeres en los resultados de la prueba SIMCE (Sistema de Medición de la Calidad de la Educación) de matemáticas, posicionan al género masculino siempre superior (Madero, 2011). Este dato no es menor al momento de hablar de las diferencias de género, en el aprendizaje de los estudiantes, en las escuelas del sistema educacional chileno. El presente artículo tiene por objetivo, analizar los discursos, de los docentes, de una escuela pública en la región del Maule, en Chile, como generadores de formas de pensamiento de género, que refuerzan las posturas hegemónicas. En tanto, la pregunta que subyace es: ¿Cuáles son los discursos de los profesores y profesoras que actúan como un reforzamiento de la diferencias de género en la escuela a través del ejercicio docente?

Respecto a la contribución del estudio, éste aportará con información respecto a esta temática, la cual ha sido escasamente investigada en la región donde se ubica el establecimiento.

Desde una mirada general Trimiño (2015), propone un sistema educativo transformador, que elimine las discriminaciones y que incorpore el pensamiento femenino, para lo cual es necesario generar más espacios para reflexionar e investigar desde la perspectiva de género. Esta situación requiere de políticas que apoyen y fomenten cambios en la apertura en la formación inicial de los profesionales docentes en materia de género.

Munevar (2011), sostiene que las presiones que se producen por los temas de género en el ámbito educacional, plantean reflexiones respecto a todo lo que implica la institucionalidad y su funcionamiento, y que contribuye a transformar la manera de trabajar los asuntos educativos en la universidad y en las posibilidades de compartir conocimientos en la escuela. Lograr que la igualdad sea efectiva en las escuelas, significa que todas las acciones que afectan la intención educativa se han de realizar desde un enfoque integrado de género, a través de un lenguaje que nombre y visibilice a todos y todas con una base democrática y paritaria. Esto hace pensar que la escuela tradicional ya no sirve, por lo que hay que avanzar hacia una pedagogía alternativa, dialógica y de género (García, 2014). Por otra parte, Poblete (2011), agrega que se debe 
hacer un trabajo transversal con todos los involucrados, además de las instituciones que participan en las decisiones educativas, profundizando en la convivencia de género rompiendo con los estereotipos arraigados en la sociedad y por ello también en la escuela.

\section{Metodología}

El presente estudio se realizó desde un enfoque cualitativo, se investigó parte de una realidad educativa, desde donde se interpreta un fenómeno que involucra personas de una forma individual y subjetiva. La concepción de la realidad entra en la perspectiva humanista, por cuanto es una investigación interpretativa, referida al individuo, a lo particular (Bisquerra, 2012).

Se propone y contextualiza la investigación cualitativa, desde la perspectiva socio educacional, como una actividad sistemática, orientada a la comprensión en profundidad de fenómenos educativos y sociales, a la transformación de prácticas, escenarios socioeducativos, a la toma de decisiones y también hacia el descubrimiento y desarrollo de un cuerpo organizado de conocimiento (Sandín, 2003).

\section{Contexto y participantes}

El estudio se llevó a cabo en un establecimiento educacional público y rural, en la comuna de San Clemente, en la región del Maule, ubicada en la zona centro sur de Chile.

Previo a su ejecución se establecieron algunos criterios de representatividad, en relación a la participación de los docentes; la totalidad de los participantes no debían superar los 6 sujetos, lo que da cuenta de presentar un diseño de estudio de caso, las edades debían fluctuar entre los 25 y 50 años, 3 de los participantes son mujeres y 3 son hombres. Finalmente, y sin criterios de exclusión, los docentes imparten las asignaturas de lenguaje, ciencias, historia, matemáticas y artes visuales.

\section{Procedimientos y técnicas}

Para efectos del presente estudio se opta por la técnica de la entrevista semi estructurada, con el fin de solicitar información a un grupo de personas, respecto a su percepción en temas de género que involucra su práctica docente (Rodríguez, Gil \& García, 1996). La técnica de la entrevista se efectúo en el establecimiento educacional donde trabaja el profesorado, los horarios y espacios fueron acordados en forma previa, sin límite de tiempo para la entrevista e informando a los participantes de la confidencialidad de la información obtenida y el propósito del estudio.

Se acordó con los participantes realizar un consentimiento informado, con el fin de resguardar absoluta confidencialidad de los antecedentes obtenidos.

El lugar en el cual se realiza la entrevista es al interior del mismo establecimiento educacional, donde trabajan los docentes, la sala de entrevista contó con las condiciones ambientales óptimas, para la ejecución del proceso. La duración de cada entrevista fluctúo entre los 45 a 60 minutos. Se proporcionó tiempo entre las preguntas para que el entrevistado solicitase pasar a la siguiente pregunta. Los horarios se organizan con cada docente de acuerdo a su disponibilidad.

Finalmente para acceder al discurso individual y colectivo de los docentes participantes se les asignó el siguiente orden: RH1-RH2-RH3 Y RM1-RM2-RM3. Esta clasificación 
presenta dos grupos divididos en hombres que se distinguen de las mujeres por las letras intermedias $\mathrm{H}$, para los profesores y $\mathrm{M}$, para las profesoras.

\section{Análisis}

El proceso de la información se realiza a través del análisis de contenido, que permite analizar en detalle y profundidad el contenido de las entrevistas (Holsti, 1968).

En primera instancia se realiza la revisión de la literatura a modo de rastreo de posibles categorías. Luego se agrupan los conceptos en subcategorías, que luego se transformaron en las categorías centrales, esta es la etapa del proceso donde se relacionaron los conceptos según rasgos específicos, o ciertas similitudes. Posteriormente, se describe todo el fenómeno comunicativo mediante la vinculación de las categorías principales.

Analizar discursos son actos lingüísticos simultáneos y secuencialmente interrelacionados, y se expresan en los ámbitos sociales y de la acción (Wodak, 2003). Así, el discurso se interpreta como un evento comunicativo completo en una situación social (Meersohn, 2005). Esto implica observar las palabras en una situación educativa comunicativa, real. Van Dijk (1989), sostiene que el discurso no sólo se encuentra en el conjunto de palabras y oraciones expresadas en el texto y el habla, sino también en las representaciones cognitivas y estrategias involucradas durante la producción $\mathrm{o}$ comprensión del discurso. Por lo tanto, en este estudio, fue posible establecer las representaciones obtenidas de los discursos de los docentes entrevistados.

En la entrevistas codificadas se establecen dos categorías centrales: igualdad de género, a la cual tributan las subcategorías el valor y respeto de los femenino y masculino y la categoría la segunda categoría central que se relaciona con la hegemonía de lo masculino, la cual contempla las subcategorías sobre la invisibilización de lo femenino. Las categorías centrales se definen como:

Igualdad de género: se expresa en tener los mismos derechos y eliminar cualquier barrera de discriminación, para participar y desarrollarse plenamente en la sociedad (Mujeres, O. N. U. 2015).

En esta categoría central tributa la categoría abierta; el valor y respeto de lo femenino.

Hegemonía de lo masculino: Connell (1987), la define como la superioridad que se enmarca en la doctrina religiosa, en la ideología política social, en el contenido de los medios de comunicación masivos, el diseño de los hogares, las políticas de bienestar de los estados, etc. La masculinidad hegemónica es el sustento del poder que se ejerce desde la superioridad masculina, asimismo implica una gran cantidad de hombres y mujeres que estén dispuestos a sostener la hegemonía, pues al no ser un dominio impuesto desde la exterioridad implica un consentimiento de parte importante de la sociedad (Connell, 1987).

En esta categoría central tributa la categoría abierta; la invisibilización de lo femenino.

Organización de las categorías obtenidas del análisis de la información:

\begin{tabular}{|l|l|l|}
\hline $\begin{array}{l}\text { Categoría } \\
\text { central }\end{array}$ & Subcategorías & Argumentos \\
\hline & & Tratando que no haya diferencias \\
\hline
\end{tabular}




\begin{tabular}{|c|c|c|}
\hline $\begin{array}{l}\text { Igualdad } \\
\text { de género }\end{array}$ & $\begin{array}{lr}\text { El valor } & y \\
\text { respeto de } & \text { lo } \\
\text { femenino } & y \\
\text { masculino } & \end{array}$ & $\begin{array}{l}\text { entre niños y niñas. } \\
\text { - Trato que todos participen de } \\
\text { igual manera. } \\
\text { - Saludamos hola alumnos, } \\
\text { alumnas. - } \\
\text { - No generando ningún tipo de } \\
\text { diferencia. } \\
\text { - En mi asignatura yo no hago } \\
\text { distinción. } \\
\text {-Las actividades están hechas para } \\
\text { los } 2 \text { géneros. } \\
\text { - Durante la clase no hago } \\
\text { diferencia. } \\
\text { - Hay que entender que la escuela } \\
\text { está compuesta por géneros de este } \\
\text { tipo, masculino y femenino. } \\
\text { - Aplicar una medida de conducta } \\
\text {-...lo aplico sea niño o sea niña. } \\
\text { - Iguales dentro de una sociedad. } \\
\text { - Generalmente dando un trato } \\
\text { igualitario. } \\
\text { - Trato de explicar igual para } \\
\text { todos. } \\
\text { - La actividad adaptada para que lo } \\
\text { puedan lograr niños y niñas. } \\
\text { - Sin distinción que los niños } \\
\text { puedan saber más que las niñas. } \\
\text { - No yo tomo el currículum como, } \\
\text { en caso de mi asignatura como } \\
\text { viene, no la modifico porque } \\
\text { vienen hechas sin diferenciar el } \\
\text { género. }\end{array}$ \\
\hline $\begin{array}{l}\text { Hegemonía } \\
\text { de lo } \\
\text { masculino }\end{array}$ & $\begin{array}{l}\text { La } \\
\text { invisibilización } \\
\text { de } \quad \text { lo } \\
\text { femenino. }\end{array}$ & $\begin{array}{l}\text { Porque cuando uno hace mucha } \\
\text { diferenciación de género ... } \\
\text { - El tema del período para ellas es } \\
\text { un problema... yo creo que es más } \\
\text { psicológico. } \\
\text {-La historia a veces es más } \\
\text { sensible para las mujeres. } \\
\text { - Cuando hay guerras, } \\
\text { matanzas...para el hombre es más } \\
\text { entretenido. } \\
\text { - Se le dice a la niña que lo haga } \\
\text { con más cuidado, pienso que ella } \\
\text { nunca trabaja mucho con esos } \\
\text { elementos, como cuchillo o } \\
\text { cartonero. } \\
\text { - Solamente el fútbol va a ser para }\end{array}$ \\
\hline
\end{tabular}




\begin{tabular}{|l|l|}
\hline & $\begin{array}{l}\text { hombres y las niñitas van a jugar } \\
\text { otro tipo de actividades. }\end{array}$ \\
- Ir al baño ..... los niños les \\
restrinjo y a las niñas les doy más. \\
Permiso al baño...cosas más \\
sensibles.
\end{tabular}

Tabla1: presenta 2 categorías centrales y 2 subcategorías, además de los argumentos que tributan para formar la clasificación producto del procesamiento de la información.

\section{Resultados}

Subcategoría: el valor y respeto de lo femenino y masculino.

En los discursos analizados, el respeto que expresan los docentes por lo femenino y masculino, se expresa en la valoración que realizan ellos mismos en sus prácticas pedagógicas.

Testimonio: (saludo).

RME: saludamos hola alumnos y alumnas.Respecto al saludo, el profesorado declara que no hace distinción de género y procuran referirse a ambos de la misma forma. Esto se evidencia al no generalizar bajo el concepto "buenos días niños", tanto a niñas y niños como un conjunto.Testimonio: (actividades en clases).

RM1: Haciendo diferentes actividades, tratando de que no haya una diferencia entre niños y niñas.

RM3: En las distintas actividades que yo hago trato de que todos participen de igual manera.

RH2: En mi asignatura yo no hago distinción.

RH3: Las actividades están hechas para que los dos géneros actúen de la misma forma.

RH2: ..., para mi hombres y mujeres dentro de la sala son iguales.

RM3: ....trato de explicar igual para todos.

RM1: ..., en los materiales que trabajo con mis niños son para todos iguales,..

RH1: .... todos se les da el mismo tiempo, la misma actividad, todo por igual.

RM3: ... sino que uno a veces observa en las clases que hay una niña que a lo mejor tu sabes que a la primera a lo mejor no va a entender con las indicaciones y te acercas y le vuelves a explicar pero también puede ocurrir en el niño, entonces uno lo hace no por diferencia del género, sino que uno lo hace porque ya conoce a los niños en la sala y más o menos su aprendizaje como va...

RH1: Generalmente dando un trato igualitario a ambos a niños y niñas, la misma cantidad de preguntas, las mismas preguntas que se le hacen a niños se le hacen a las niñas, el mismo tiempo que se le da para una actividad a una niña es el mismo tiempo para un alumno enfocando siempre el trato por igual a ambos se le utilizan las mismas palabras...

RH3: No yo tomo el currículum como, en caso de mi asignatura como viene, no la modifico porque vienen hechas sin diferenciar el género....

RH2: En cuanto a mis practicas pedagógicas van netamente enfocada para hombres y mujeres sin distinción de que los niños pueden saber más que las niñas o viceversa, sino que siempre mirados por igualdad.En relación a las actividades en clases, los docentes 
procuran mantener la igualdad entre niños y niñas, desde donde se refleje la participación sin diferencias. Consideran que las actividades no requieren ser modificadas al argumentar que los niños y niñas logran aprender indistintamente. En tanto, si existe un problema éste no se debe a un problema de género sino a algún posible problema de aprendizaje. En cuanto a las explicaciones que realiza el docente sobre una actividad académica y la participación de los alumnos y alumnas en una tarea, no existe diferencia de género ya éstos enfocan el trabajo para que los estudiantes participen por igual. Sin embargo, existe la posibilidad que el profesorado no necesariamente se focalice en la igualdad de género hacia sus estudiantes, sino que es probable que se limiten a dar sólo una explicación, para la ejecución de una tarea, como también crear una actividad académica estándar para alumnos y alumnas.

Testimonios:

RM3: ...niños pasa de que yo llamo la atención sea niño sea niña yo le llamo la atención igual y por ejemplo si tengo que aplicar una medida de conducta lo aplico sea niño o sea niña, o sea todos los que están involucrados....

Respecto a situaciones externas a la sala de clase, se desprende del quehacer docente, que si bien existen problemas de conductas, las sanciones no hacen referencia al género, por lo tanto, se entiende que las alumnas y alumnos pueden generar conductas disruptivas en algún momento de la rutina escolar y los castigos y amonestaciones son en función de la gravedad de la falta. Por ello, es preciso aclarar que para efecto de este estudio, las situaciones conductuales no se relacionan con el ser hombre o ser mujer.

Testimonio:

RM1: Las expectativas que tengo con los niños es que a temprana edad la mayoría de edad sean profesionales, que sean muy felices y que se sientan aceptados y que sean iguales dentro de una sociedad.

RM2: Yo espero que todos sean profesionales, que todos logren y bueno si no son profesionales que sean los mejores en lo que quieran hacer ellos. Que sean felices y que logren todo lo que ellos quieran no importa que sean hombres o mujeres, pero que logren sus objetivos de vida, ya sea en un técnico en un profesional en lo que quieran hacer.

RM3: Bueno yo trabajo de quinto a octavo y yo tengo alta, o sea muchas expectativas con los niños, o sea sé que tenemos en los cursos niños bastante buenos que pueden llegar muy lejos...

RH1: Las expectativas generalmente son, que manejo yo con los alumnos son altas.

RH2: Bueno vuelvo a repetir el tema para mi niños y niñas son iguales y lo que yo hago en clases siempre los motivos a que sigan estudiando, que ellos pueden, trato de siempre motivarlos y sin distinción de género.

RH3: Como género que como alumnos que los dos obtengan un mismo nivel de educación, que los dos salgan adelante y sean en su futuro unas excelentes personas.

Los argumentos anteriores ilustran las expectativas que muestran los docentes frente a sus estudiantes. Manifiestan no hacer distinción cuando se habla de proyectar el futuro académico y desarrollo profesional de sus alumnos y alumnas. No se limitan sólo a un plano académico, sino también al desarrollo de los aspectos sociales y de vida. Por otra parte, reconocen las capacidades y potencialidades de sus estudiantes al momento de realizar su quehacer profesional. Este reconocimiento se produce desde temprana edad escolar y aparentemente no varía en tanto avancen en su recorrido escolar.

Los docentes manifiestan no discriminar, ni invisibilizar a las niñas respecto al logro de objetivos para la vida laboral y profesional. Las expectativas que se forman en cuanto a 
sus capacidades no hacen referencia al ser hombre o mujer. La gran mayoría de los docentes entrevistados esperan lo mejor para sus alumnos y alumnas, aunque se denota una invisibilización del género al utilizar "ellos" para referirse tanto a niñas como a niños.

\section{Categoría abierta: la invisibilización de lo femenino.}

Testimonio:

RH1: ....en el caso de la historia a veces se hablan temas un poco más fuertes: guerras, muertes, torturas, sobre todo en la historia antigua o la historia mapuche que para las mujeres a veces es más sensible, que para las mujeres a las niñas les cuesta un poco más entenderlo asimilarlo y les afecta un poco. Por ejemplo cuando hay guerras, matanzas, videos, películas, al hombre para el hombre es más entretenido es más motivante para la mujer le afecta un poco y ahí hay que hacer una diferencia al momento de explicar, dar una explicación que en ambos casos puedan el hombre el niño salga de lo entretenido y la mujer salga de lo sensible para que puedan entender más el proceso fríamente, críticamente.....

RH3: ...hay que agudizar los palos entonces se le dice a la niña que lo haga con más cuidado porque puede ser medio peligroso, porque pienso que ella nunca trabaja mucho con esos elementos, como cuchillos o cartonero.

RM2: Trato de adaptar actividades que sean como de gusto de las niñas y gusto de los niños, como por ejemplo cuando ellos van a hacer una obra dramática trato de darles como posibilidades por ejemplo de las princesas o cosas así sobre todo a los más chicos de quinto y a los más grandes de cars de eso a los hombres porque eso le gusta más o de súper héroes.

Los discursos analizados y categorizados en la invisibilización de la mujer, dan cuenta que en algunos aspectos de las actividades lectivas los docentes presentan subestimación hacia las niñas. Consideran que la información relacionada con violencia o fuerza es propia de los hombres. En tanto, existe el ideario que las niñas son más democráticas, maduras y captan los temas valóricos con mayor ventaja en relación a los hombres.

Por otra parte, los docentes encasillan a las niñas en estereotipos vinculados a las princesas, relacionas a personalidades de bajo perfil, calladas, obedientes, arregladas, sumisas y domésticas, entre otros aspectos. Por el contrario, los hombres, se relacionan con la fuerza, la valentía, el poder y la decisión. Esta forma de categorizar a los estudiantes no hace más que reproducir y aumentar las diferencias con características sexistas.

Testimonio:

RM1: ... solamente el futbol va a ser para hombres y las niñitas van a jugar otro tipo de actividades que a lo mejor sea menos violento....

RH1: ... por ejemplo se sigue jugando por ejemplo a la cuerda, que van saltando la cuerda y hay puras niñas, van a jugar a la pelota, puros niños, y a veces al juego tradicional "pillarse".

En cuanto a las actividades de juego en la escuela, los docentes manifiestan que existen evidentes diferencias en las actividades lúdicas que realizan los estudiantes en horas no lectivas, como en recreos y talleres extra programáticos. Consideran el encasillamiento de los deportes según corresponda a las niñas y otros a los niños. Nuevamente los estereotipos salen a la luz, relacionan el riesgo y la violencia en el juego con los 
hombres, en tanto vinculan actividades lúdicas menos arriesgadas a las niñas, donde la integridad física no sea expuesta.

Testimonio:

RM2: ..igual hay muchas cosas que en una mujer son mal vistas, hay muchas cosas que no se pueden hacer, como por ejemplo no sé si ven a una niña que va constantemente a la plaza es porque es loca, porque aquí porque allá, como es un pueblo chico todos nos conocemos entonces eso igual afecta o si ven a una niña por ejemplo que tiene no sé séptimo, octavo y ha tenido dos o tres pololos, ah ya es pero como lo peor, como que nadie va a querer pololear con ella...., en cambio los niños no, los niños si uno ve por ejemplo un niño de octavo en una plaza a las dos o tres de la mañana ah no es tanto total es hombre.

Respecto a la cultura local, los docentes manifiestan cierto prejuicio hacia las conductas de las niñas adolescentes en su vida complementaria a la escuela. Sus comportamientos sociales son enjuiciados por la tradición conservadora y moralista de los habitantes de la comunidad por lo que deben asumir las consecuencias tan sólo por el hecho de ser mujer. Al hombre, en tanto el patrón cultural abala sus conductas, se le permite todo tipo de vicios, conductas y lenguaje por su condición sin riesgo de ser cuestionado. Esto da cuenta de la cultura dominante en un contexto específico que se genera de los discursos dominantes que influencia el conocimiento social compartido, actitudes e ideologías (Van Dijk 1993).

Fries \& Matus (1999), sostienen que la cultura del patriarcado se relaciona a la experiencia masculina y es asumida como experiencia humana dando lugar a un sistema de dominación jerarquizada hacia la oposición masculina.

La escuela en tanto, no queda fuera del contexto cultural de la comunidad. Asimismo es posible encontrar similares prejuicios al interior de las salas de clases, desde donde se comienza a encasillar a las niñas con un comportamiento moralmente estricto, mientras que toda conducta fuera de lo culturalmente permitido se considera incorrecta. Esto lleva a inferir que las conductas de los niños son permitidas sin dar mayor relevancia al margen que estas puedan ser agresivas.

Testimonio:

RM2: ...por ejemplo influye en el tema de las niñas sobre todo los cursos más grandes, el tema de su periodo, para ellas es un problema y necesitan constantemente estar yendo al baño y yo creo que es más psicológico de ellas.

RM2: Eso sí por ejemplo a los niños les restrinjo más el permiso, y a las niñas les doy más, eso es como lo más notorio que yo hago.

RH2: Bueno lo mismo a la anterior, pero yo creo que al final igual uno como que hace distinción en algunas cosas en algunos detalles como por ejemplo que no son netamente pedagógicas, sino que son más bien, más personales como permiso al baño, que cosas más sensibles, no se voy por ese lado.

Los docentes fundamentan sus decisiones sobre temas no pedagógicos en función a ser hombres o mujeres. Atribuyen la autoridad para generar un permiso a diferencias biológicas y o psicológicas que están a la base de diferencias de ser niña o niño. Esto habla de aspectos y diferencias que los docentes han internalizado y llevan a la práctica en forma constante sin reflexionar sobre el aumento de la desigualdad.

Testimonio:

RH2: Generalmente no hago mucha diferencia.

RM1: Porque cuando uno hace mucha diferenciación de género. 
RM3: Nosotros marginamos al niño que es más inquieto.

RM1: Generalmente los docentes hacemos la diferencia de siempre separar a los niños de las niñas.

RH2: ..., yo creo que depende igual netamente del profesor que está en la clase si es que hace distinción o no y los niños lo perciben.

Los docentes argumentan tener claridad al momento de realizar diferencias de género, las que manifiestan en forma explícita y directa en sus alumnos y alumnas. El profesorado tiene plena conciencia de realizar diferenciación a través de sus prácticas pedagógicas y que esta puede ser gradual dependiendo del tipo de diferencia que pretenda acentuar. Beneficia o perjudica al niño o niña según su creencia y prejuicio, sin importar si el individuo tiene conocimiento de aquello. Por ello, es posible afirmar la existencia de actitudes de indiferencia del docente hacia el estudiante que está en formación. Es probable que el docente no esté consiente del grado de replicabilidad que puede provocar en sus estudiantes en un tiempo inmediato o a futuro.

\section{Conclusiones}

La categoría central de igualdad de género, evidencia que los docentes intentan responder a los lineamientos que establece el Ministerio de Educación, a través de la implementación de políticas y programas orientados a superar las diferencias entre niñas y niños.

El profesorado tiene plena conciencia que los estudiantes deben tener las mismas oportunidades frente al aprendizaje, sin exclusiones ni asociaciones imaginarias que predispongan a los niños o niñas a desarrollar y potenciar ciertas habilidades, en tanto otras se vean disminuidas por el hecho de ser hombre o mujer. Sin embargo, no queda claro si al momento de explicar algún aspecto del currículum o proponer una actividad lectiva los docentes intentan no hacer diferencias o bien utilizan estrategias estandarizadas para la enseñanza.

Respecto a la segunda categoría central sobre la hegemonía de lo masculino, es posible concluir que existe predominio de la invisibilización de lo femenino en aspectos complementarios al curriculum. Estos se caracterizan por ser de índole cultural y social, los que emerger producto de la convivencia, específicamente en actividades propias del desarrollo de los estudiantes y que involucran aspectos personales y o bilógico.

Otro aspecto relevante, es el hecho que los docentes son portadores de replicar las diferencias de género en forma inconsciente al promocionar la desigualdad hacia las niñas en relación a los niños. Se expresan en la verbalización de limitantes respecto a sus capacidades para ejecutar tareas propias del currículum producto de una idea proteccionista y maternal. Por otra parte, los estereotipos a través de los cuales los docentes encasillan a los alumnos y alumnas limitan el desarrollo de sus potencialidades estimulando aquellas que son propias y generadoras de las diferencias, (princesas y superhéroes). Cada una de las cuales trae consigo una carga social que vincula ambos conceptos con ideales y fantasías que alejan a los estudiantes de la realidad. Esto puede llevar a los estudiantes a tener una visión parcial en lo que se refiere a la vida social y afectiva, debido a ideas poco reales y alejadas de su contexto. Finalmente, es importante precisar la necesidad que los docentes profundicen en temas relativos al género, para erradicar las desigualdades y discriminación que en muchas ocasiones perjudican y limitan el desarrollo de la mujer y del hombre. 


\section{BIBLIOGRAFÍA}

ARNOT, M. (2009). Una conciencia global? La incorporación de las injusticias de género en la educación global ciudadanía. Educación, Ciudadanía y justicia Social, 4 (2), 117-132.

BAS, E., PÉREZ. V. \& VARGAS, M. (2014). Contribución de la lectura a la formación de género, en el Grado de Educación Social: Estudio descriptivo y censal Ocnos.

BISQUERRA, R. (2012). Metodología de la investigación educativa. Madrid: La Muralla.

BOLÍVAR, A. (2015). Justicia social y equidad escolar. Una revisión actual.Revista Internacional de Educación para la Justicia Social, 1(1).

BORRIES, V. (2012). Reflexionando en torno al concepto de transversalización de génro. Delineando una tipología para el análisis de sus expresiones empíricas. Punto Género. 2 (20), 11.

CAMERON, D. (1992). Feminism and linguistic theory. Londres: Macmillan.

CENTRO DE DOCUMENTACIÓN E INVESTIGACIÓN EDUCATIVA (1992). Las desigualdades en la Educación en España. Madrid. CIDE, Ministerio de Educación y Ciencia.

CONNELL, R. W. (1987). Gender and power: Society, the person and sexual politics. Cambridge: Polity Press.

CORTÉS, R. J. (2007). Discurso de género y práctica docente. Revista de Investigación Educativa, 25(1), 59-76.

DA SILVA, D., FANFA, D. Y FOSSATTI, P. (2012). Género y sexualidad: ¿Qué dicen las profesoras de educación infantil de Canoas, Brasil? Archivos Analiticos de Políticas Educativas, 20 (16), 1-21.

EGAÑA, M., NÚÑEZ, I. \& SALINAS, C. (2003). La educación primaria en Chile: 1860 - 1930. Una aventura de niñas y maestras. Santiago: Lom ediciones.

FINCO, D. (2015). Igualdad de género en las instituciones educativas de la primera infancia brasileña. Latinoamericana de Ciencias Sociales, Niñez y Juventud, 13 (1), 85-96.

FOUCAULT, M. (1970). El orden del discurso. Barcelona, Tusquest. 1987.

FRIES, L. \& MATUS, V. (1999). "Supuestos ideológicos, mecánicos e hitos fundantes del derecho patriarcal": En A. Facio \& L. Fries. $\left(1^{\mathrm{a}}\right)$, Género y derecho (143-148). Santiago, Chile: Lom.

GARAY, A., IÑIGUEZ, L. \& MARTÍNEZ, M. (2005) La perspectiva discursiva en psicología social. Subjetividad y procesos cognitivos, (7), 105-130.

GARCÍA, M. (2014). Prácticas de género invisibles en la escuela. Cuadernos de pedagogía, (441), 97-100.

GARCÍA, R., REBOLLEDO, M., GARCÍA, R., GONZÁLEZ, R., BARRAGÁN, B. Y

RUIZ, E. (2010). Actitudes del alumnado hacia la igualdad de género. Investigación educativa, 28 (1), 217-232.

GILBERT, G. \& MULKAY, M. (1984). Opening Pandora `s Box: A Sociological Análisis of Scientist 'Discourse. Cambridge, Cambridge University Press.

HOLSTI, R. (1968): Content analysis. En Lindzey, G. y Aronson, E. The 
handbook of social Psychology. Vol 2. Research Methods, Addison-Wesley, Reading, Mass.

INSTITUTO NACIONAL DE DERECHOS HUMANOS.

(2012). Informe: Comité para la eliminación de la Discriminación contra la Mujer. 2012. Santiago de Chile, 2012.

IÑIGUEZ, L. \& ANTAKI, CH. (1994) "El análisis del discurso en

Psicología Social”, Boletín de Psicología, Vol 44, Pág. 57-75.

JIMÉNEZ, R. (2007). Discurso de género y práctica docente. Revista de educación educativa, 25 (1), 59-76.

LAKOFF, R. (1975). Language and women `s place. Nueva York: Harper Colophon [Trad Cast. El lenguaje y el lugar de la mujer. Barcelona: Hacer, $3^{\mathrm{a}}$ edición, 1995].

MADERO, I. (2011). Inclusión y exclusión de género y clase al interior de la escuela chilena en 4 comunas del sur de Chile. Estudios Pedagógicos, 37 (2), 135-147.

MADRID, P. (2011). Masculinidades y equidadde género en la escuela: consideraciones para la construcción de una política educativa en Chile. En F. Aguayo. M. Sandler. Masculinidades y Politicas Públicas. Involucrando hombres en la equidad de género. Chile: LOM.

MEERSOHN, C. (2005)Introducción a Teun Van Dijk: Análisis de discurso. Revista Cinta de Moebio 24 (s.d.)

MUNEVAR, D. (2011). Los saberes de género en tesis que investigan asuntos educativos. Latinoamericana de educación inclusiva, 5 (1), 91- 105.

MUJERES, O. N. U. (2015). El progreso de las mujeres en el mundo 2015-16. Transformar las economías para realizar los derechos. op. cit.

PARGA, L. (2011). Las rutas de inserción a la docencia en educación secundaria. Una mirada de género. Latinoamericana de educación inclusiva, 5 (1), $49-52$.

POBLETE, R. (2011). Género y educación: trayectorias de vida para ellos y ellas. Revista latinoamericana de educación inclusiva, 5 (1), 63-77

RODRÍGUEZ, C. (2011). Género y cultura escolar. Madrid. España: Morata.

RODRÍGUEZ, G., GIL, F. \& GARCÍA, E. (1996). Metodología de la investigación cualitativa. Málaga: Aljibe.

SAFILOS - ROTHSCHILD, C. (1987). Las diferencias según el sexo en la socialización y la educación infantil y sus consecuencias en la elección de los estudios y sus resultados. En OCDE La educación de lo femenino. Estudio internacional sobre las desigualdades entre muchachas en la educación. Barcelona. Aliorna, pág. 43 - 80.

SÁNCHEZ, M. (2015). Unidad de trabajo educativo en género.(Maestría en pedagogía del sujeto).

SANDÍN, M. (2003). Investigación cualitativa en Educación. Fundamentos y tradiciones. Madrid: McGrawHill.

TRIMIÑO, C. (2015). Género (s) y educación: Aproximaciones al contexto de América Latina y el Caribe. Historia de la Educación Latinoamericana, 17 (24), 55-72.

VARGAS, I. (2011). Reflexiones en torno a la vinculación de la 
educación y el género. Educare, 15 (1), 137-147

VAN DIJK, T. (2005). Estructuras y funciones del discurso. Ciudad de México DF, México: Siglo xxi editores.

VAN DIJK, T. (1989). Social cognition and discurse. En: H. Giles \& R.P.

Robinson (Eds), Handbook of social psychology and lenguaje. (pp.163-183).

Chichester:Wiley.

VAN DIJK, T. (1993). "Principles of critical discourse análisis". Discourse \& Society 4(2), 249-283.

VALDÉS, T. (2013). Género en la escuela o la porfiada desigualdad. Reflexiones Pedagógicas, (49), 48

VALDÉS, T. (2013). La CEDAW y el Estado de Chile: viejas y nuevas deudas con la igualdad de género. Anuario de derechos humanos, (9), 171-181.

VENEGAS, M. (2010). La igualdad de género en la escuela. Asociación de Sociología de la Educación, 3 (3), 388-402.

West, C., Lazar, M. \& Kramarae, C. (2000): El género en el discurso. En T. A. Van Dijk, (Com). El discurso como interacción social. Barcelona: Gedisa, $179-172$.

WODAK, R. (2003). De qué trata el análisis crítico del discurso (ACD). Resumen de su historia, sus conceptos fundamentales y sus desarrollos. En Wodak, Ruth y Meyer, Michel. Métodos de análisis crítico del discurso. España: Gedisa, 17-34.a. 